Results. Adult studies: In short-term studies, TEM rates were comparable in patients treated with lurasidone monotherapy 20 $60 \mathrm{mg} / \mathrm{d}(3.7 \%)$ and $80-120 \mathrm{mg} / \mathrm{d}$ (1.9\%) vs. placebo (1.9\%). TEM rates were also comparable in patients treated with lurasidone 20$120 \mathrm{mg} / \mathrm{d}(1.1 \%)$ adjunctive to Li/VPA vs. placebo + Li/VPA (1.2\%). In the monotherapy study, significant reduction in YMRS score was observed at study endpoint for the $20-60 \mathrm{mg} / \mathrm{d}$ group compared to placebo $(-1.9$ vs. $-1.3 ; \mathrm{p}<0.05)$ with similar improvement relative to placebo in the $80-120 \mathrm{mg} / \mathrm{d}$ group. Change for YMRS score was comparable for lurasidone and placebo in the adjunctive study. In long-term studies, $1.3 \%$ of adult patients treated with lurasidone monotherapy $(n=316)$ met criteria for mania, and $3.8 \%$ of patients on adjunctive lurasidone therapy $(\mathrm{n}=497)$ met TEM criteria. Pediatric studies: TEM rates were comparable in patients treated with lurasidone vs. placebo $(1.7 \%$ vs. 2.3\%). LS mean reduction in symptoms of mania from baseline to week 6 was significantly greater for lurasidone vs. placebo on YMRS score $(-2.0$ vs. $-1.1 ; \mathrm{p}<0.05)$. Pediatric long-term studies: After two years of OL treatment with lurasidone, $5.2 \%$ of patients met TEM criteria. Mean change in YMRS total score from DB baseline to Month 24 continued to improve $(-2.0)$.

Conclusions. Short-term and long-term treatment with lurasidone demonstrated significant improvement in manic symptoms and was not associated with an increased risk of TEM in either adult or pediatric patient populations compared to rates reported in clinical populations of patients.

Funding. Sunovion Pharmaceuticals Inc.

\section{Long-Term Effectiveness of Lurasidone in Pediatric Bipolar Depression: Response, Remission and Recovery}

\section{Manpreet Singh, $\mathrm{MD}^{1}$, Michael Tocco, $\mathrm{PhD}^{2}$, Edward Schweizer, $\mathrm{MD}^{3}$ and Andrei Pikalov, MD, $\mathrm{PhD}^{2}$}

${ }^{1}$ Stanford University, Stanford Pediatric Mood Disorders Program, Stanford, CA, USA, ${ }^{2}$ Sunovion Pharmaceuticals Inc., Marlborough, MA, USA, and ${ }^{3}$ Paladin Consulting Group, Princeton, NJ, USA

Presenting Author: Michael Tocco response or remission in children and adolescents with bipolar depression followed over a two-year period.

Method. Patients 10-17 years with bipolar I depression who completed a 6-week double-blind (DB) study of lurasidone vs. placebo were eligible to enroll in a two-year, open-label (OL) extension study in which patients were continued on flexibly-dosed lurasidone (20-80 mg/d) or switched from placebo to lurasidone. Efficacy measures included the Children's Depression Rating Scale, Revised (CDRS-R) and the Clinical Global Impression, Bipolar Depression Severity scale (CGI-BP-S). Functioning was evaluated utilizing the Clinician-rated Children's Global Assessment Scale (CGAS) score, with a score $>70$ indicating no clinically meaningful functional impairment. Responder criteria were met if a patient achieved criteria $=50 \%$ reduction from $\mathrm{DB}$ baseline in the CDRS-R total score: remission criteria were met if a patient achieved a CDRS-R Total Score $=28$ and a YMRS total score $=8$ and CGI-BP-S depression score $=3$, and a patient was considered to have met recovery criteria if they achieved remission with a CGAS score $>70$. In addition, a more stringent outcome, sustained remission, was also analyzed, which required a patient to meet remission criteria for $=24$ consecutive weeks.

Results. A total of 306 patients completed the 6-week DB study and entered the extension study; 195 (63.7\%) patients completed one year of treatment and 168 (54.9\%) patients completed two years of treatment. Responder rates at OL baseline, one year, and two years were: $51.0 \%, 88.4 \%$ and $91.1 \%$, respectively; remission rates were $24.3 \%, 61.3 \%$, and $75.6 \%$, respectively; and recovery rates were $17.7 \%, 53.8 \%$, and $73.8 \%$. On a Pearson correlation analysis, there was a strong inverse relationship $(r=-0.71)$ between CDRS-R total score, and global functioning as measured by the CGAS. Sustained remission was achieved by $37.2 \%$ of patients at one year and $57 \%$ of patients after two years. Conclusions. In children and adolescents with bipolar depression, up to 2 years of treatment with lurasidone was associated with continued improvement in depressive symptoms, resulting in progressively higher rates of response, remission, recovery, and the more rigorously calculated outcome of sustained remission. Funding. Sunovion Pharmaceuticals Inc.

\title{
Safety and Effectiveness of SEP-363856 in Schizophrenia: Results of a 6-Month, Open-Label Extension Study
}

Christoph U. Correll, $\mathrm{MD}^{1}$, Kenneth S. Koblan, $\mathrm{PhD}^{2}$, Seth C. Hopkins, $\mathrm{PhD}^{2}$, Justine Kent, $\mathrm{MD}^{2}$, Hailong Cheng, $\mathrm{PhD}^{2}$, Robert Goldman, $\mathrm{PhD}^{2}$ and Antony Loebel, MD ${ }^{2}$

${ }^{1}$ The Zucker Hillside Hospital, Department of Psychiatry, Northwell Health, Glen Oaks, NY, USA; Hofstra Northwell School of Medicine, Department of Psychiatry and Molecular Medicine, Hempstead, NY, USA; and Charité Universitat Medizin, Department of Child and Adolescent Psychiatry, Berlin, Germany, and ${ }^{2}$ Sunovion Pharmaceuticals Inc., Marlborough, MA, USA

Presenting Author: Kenneth S. Koblan 